\title{
Is calibration of the perception of length modality-independent?
}

\author{
Jeffrey B. Wagman • Drew H. Abney
}

Published online: 31 May 2013

(C) Psychonomic Society, Inc. 2013

\begin{abstract}
Perception becomes increasingly scaled to environmental properties when feedback is provided about the perception of a given property. Understanding the process of calibration requires a description of the stimulation patterns to which perceivers become calibrated as a result of such feedback. Recent investigations using a transfer-ofcalibration paradigm have shown that recalibration of the perception of length transfers from audition to touch. Such results have provided preliminary evidence that calibration of this property is modality-independent (i.e., that feedback about length calibrates perceivers to a modality-independent stimulation pattern). The experiment reported here provides a stronger test of this claim by demonstrating that perception transfers in both directions (i.e., from audition to touch, and vice versa). The results provide further evidence that calibration is modality-independent, and they are consistent with the more general proposal that the stimulation patterns that support perception are also modality-independent.
\end{abstract}

\section{Keywords Audition $\cdot$ Haptics $\cdot$ Perceptual learning}

Successfully performing a given behavior requires that perception be scaled to environmental properties. The process that establishes and maintains this scaling relationship is known as calibration (see Withagen \& Michaels, 2005). Calibration of perception typically requires experiences in addition to the perceptual task (e.g., feedback about performance). Among the issues that are important in understanding calibration is establishing what perceivers become calibrated to as a result of such experiences (Withagen \& Michaels, 2004). What is the nature of the stimulation pattern(s) to which participants become calibrated as a result of feedback? This question is related to (and perhaps dependent upon) a broader and theoretically important question

J. B. Wagman $(\bowtie) \cdot$ D. H. Abney

Department of Psychology, Illinois State University,

Campus Box 4620, Normal, IL 61790-4620, USA

e-mail: JeffreyWagman@ilstu.edu about perception-what is the nature of the stimulation patterns that support perception in general? This experiment focused on the former question, but has relevance to the latter, as well.

One method of elucidating what perceivers become calibrated to as a result of feedback is to investigate the circumstances under which calibration transfers across contexts (see Rieser, Pick, Ashmead, \& Garing, 1995). Many such investigations have been motivated by the observation that perception often exhibits anatomical independence (Gibson, 1966). That is, different properties can be perceived by the same anatomical components, and a given property can be perceived by different anatomical components. For example, many properties of an object can be perceived when that object is wielded by hand (Turvey \& Carello, 2011), and a given property (e.g., length) can be perceived when an object is wielded by hand, by foot, or by torso (Hajnal, Fonseca, Harrison, Kinsella-Shaw, \& Carello, 2007; Palatinus, Carello, \& Turvey, 2011). Such anatomical independence of perception suggests that calibration of perception may also be anatomically independent. If so, recalibration of the perception of a given property should transfer from one anatomical component to another. Recent studies have shown that this is the case. For example, recalibration of perception of the length of a wielded object transfers from one hand to the other (Withagen \& Michaels, 2004) and from hand to foot (Stephen \& Hajnal, 2011).

Given that different perceptual modalities generally consist of different anatomical components, modality independence may be a special case of anatomical independence (Wagman \& Abney, 2012). Along these lines, different properties can be perceived by the same modality, and a given property can be perceived by different modalities. For example, many surface properties can be perceived by listening to sounds produced or affected by that surface (Gordon \& Rosenblum, 2004; Rosenblum \& Robart, 2007), and a given surface property (e.g., whether it is "stand-on-able") can be perceived by probing or viewing that surface (Fitzpatrick, Carello, Schmidt, \& Corey, 1994). 
Such findings are consistent with the proposal that the stimulation patterns that support the perception of a given property are also modality-independent (i.e., that they are not specific to a particular modality; Rosenblum \& Gordon, 2001). If so, then the stimulation pattern(s) to which participants become calibrated as a result of feedback should also be modality-independent, and recalibration of the perception of a given property should transfer from one modality to another. Recently, we (Wagman \& Abney, 2012) found that recalibration of the perception of object length transferred from audition (i.e., listening to an object strike the floor) to touch (i.e., wielding an object by hand). Such results have provided preliminary evidence that calibration of perception is modality-independent. A stronger test of this hypothesis would require demonstrating transfer of recalibration of perception in both directions.

\section{The present experiment}

In this experiment, we investigated whether recalibration of perception of length transfers from audition to touch, as well as from touch to audition. We chose this property and these modalities for two reasons. First, length is perceivable by both modalities, and in each case, perceived length is linearly related to actual length. This relationship allows for an analysis of calibration (and transfer of recalibration) using regression statistics (Cabe \& Wagman, 2010). Second, there is evidence that, in each modality, the perception of length is supported by analogous, modality-neutral stimulation pattern(s) (Carello, Anderson, \& Peck, 1998).

The experiment consisted of a pretest, practice, and a posttest. In the pretest, participants perceived the lengths of occluded rods by listening to those rods strike the floor and by wielding those rods about the wrist (on separate blocks of trials). During practice, participants performed only one of these tasks. Half of the participants received inflated feedback (that the rod was 1.5 times its actual length) after every practice trial, and half did not receive any feedback. The inflated feedback was provided because (1) it guaranteed (relatively) poor calibration to length in the pretest, and thus provided potential for recalibration during practice, and (2) doing so aided in avoiding a conflation of recalibration with improvements in accuracy or with changes due to repeated perceptual experience (Stephen \& Hajnal, 2011).

Given that recalibration of perception typically requires experiences in addition to the perceptual task, we expected that recalibration would occur only when feedback was provided during practice. Moreover, given (1) the hypotheses that perception and calibration are modality-independent and (2) evidence that recalibration of the perception of length transfers from audition to touch, we expected that recalibration would transfer in both directions.

\section{Method}

Participants

A group of 58 right-handed Illinois State University students $\left(53\right.$ women, five men $\left.^{1}\right)$ participated.

Materials and apparatus

Ten wooden rods $(1.2-\mathrm{cm}$ diameter; $30 \mathrm{~cm}$ to $120 \mathrm{~cm}$ in length, in 10-cm increments) were used as stimuli. Each participant sat in a right-handed student desk. A curtain occluded both a participant's hand and the rod. Participants adjusted the distance of a marker along a $240-\mathrm{cm}$ track, such that the distance between the marker and the zero point corresponded to the perceived length of the rod.

\section{Design}

During the pretest, all participants perceived the length of each rod by both touch and audition (during separate blocks). During practice, half of the participants perceived length by touch only, and half did so by audition only. Within each of these groups, participants received either inflated feedback or no feedback after each practice trial. The posttest was identical to the pretest. In the experiment, we used a 2 (test: pre vs. post) $\times 2$ (test modality: touch vs. audition $) \times 2$ (practice modality: touch vs. audition) $\times$ feedback (inflated vs. no feedback) design. Test and test modality were within-participants variables, and practice modality and feedback were between-participants variables.

\section{Procedure}

Pretest Each participant placed his or her right arm on the armrest of the desk and the right hand through a curtain, such that the wrist was aligned with the zero point of the apparatus. On a given trial in the touch condition, the participant was handed a rod so that one end was flush with the bottom of the fist. The participant wielded the rod about the wrist and reported the perceived length, as described above. Wielding was unrestricted, except that the participant was instructed not to lift the forearm off of the desk or touch the curtain with the object. On a given trial in the audition condition, the experimenter centered a rod on the edge of a ( $23 \mathrm{~cm}$ tall $\times 29 \mathrm{~cm}$ deep $\times 44 \mathrm{~cm}$ wide) cardboard box and rolled it such that it fell on to a wooden surface. The participant listened to the rod strike the surface and then reported perceived length as described above.

\footnotetext{
${ }^{1}$ Although individual differences in the perception of length by dynamic touch do exist (Withagen \& van Wermeskerken, 2009), there is little reason to suspect that such differences are gender-based.
} 
A participant could wield the rod as long as necessary, listen to a rod as many times as necessary, and continually adjust their perceptual report. After reporting perceived length on a given trial, the participant returned the marker to the zero point. Each participant perceived the length of each rod once in each modality condition. Blocks of five touch trials were alternated with blocks of five audition trials. The participant was not informed that the same rod set was used in both modality conditions. The order of modality conditions was randomized across participants, and the order of rod lengths was randomized within a given modality condition.

Practice Following the pretest, the participants in all conditions completed a practice session using a subset of the pretest rods in the following order: 30,100, 120, 50, 40, and $110 \mathrm{~cm}$ (cf. Wagman \& Abney, 2012). Participants in the touch condition perceived length by touch only, and participants in the audition condition did so by audition only. For participants in the inflated-feedback condition, feedback was provided after every trial by the experimenter repositioning the marker, such that the distance between the marker and the zero point was 1.5 times the actual length of the rod. Participants in the no-feedback condition did not receive any feedback.

Posttest The posttest was identical to the pretest.

\section{Results}

To investigate whether perceived length changed from pretest to posttest, we divided the posttest perceived lengths by the pretest perceived lengths and compared these ratio values in a 2 (test modality: touch vs. audition) $\times 2$ (practice modality: touch vs. audition) $\times 2$ (feedback: inflated feedback vs. no feedback) mixed-design analysis of variance (ANOVA). A main effect of feedback $[F(1,54)=34.70$, $p<.001, \eta_{\mathrm{p}}{ }^{2}=.39$ ] showed that ratios were larger (i.e., that the change from pretest to posttest was larger) in the inflated-feedback condition $(M=1.61, S D=0.47)$ than in the no-feedback condition $(M=1.10, S D=0.26)$. A main effect of test modality $\left[F(1,54)=26.32, p<.001, \eta_{\mathrm{p}}{ }^{2}=.32\right]$ showed that ratios were larger in audition $(M=1.47, S D=0.52)$ than in touch $(M=1.25, S D=0.36)$. Follow-up $t$ tests with Bonferroni correction confirmed that the ratios were greater than 1.0 (perceived length increased from pretest to posttest) in all conditions when feedback was provided (all $p \mathrm{~s}<.001$ ) and did not differ from 1.0 (perceived length did not change from pretest to posttest) in any condition without feedback (all $p \mathrm{~s}>.05$ ). In short, perceived length increased from pretest to posttest when feedback was provided (regardless of the test and training modalities) but did not change when feedback was not provided.
However, such analyses are not informative as to whether the increases in perceived length following feedback were due to changes in scaling (i.e., the mapping between perceived and actual values), changes in offset (i.e., a consistent tendency to over- or underestimate), or both. To examine these possibilities, we computed regression lines, with perceived length as the dependent variable and actual length as the independent variable for each participant for each modality in the pretest and posttest. Changes in the slopes and intercepts of these lines from pretest to posttest were used to quantify any changes in scaling and offset, respectively (cf. Cabe \& Wagman, 2010).

A 2 (test: pre vs. post) $\times 2$ (test modality: touch vs. audition) $\times 2$ (practice modality: touch vs. audition) $\times 2$ (feedback: inflated feedback vs. no feedback) mixed-design ANOVA was performed on the slopes of the regression lines. A main effect of test modality $\left[F(1,54)=63.61, p<.001, \eta_{\mathrm{p}}{ }^{2}=.54\right]$ showed that the slopes were larger in touch $(M=0.87, S D=0.34)$ than in audition $(M=0.59, S D=0.35)$. A main effect of test $\left[F(1,54)=54.65, p<.001, \eta_{\mathrm{p}}{ }^{2}=.50\right]$ showed that slopes increased from pretest $(M=0.61, S D=0.31)$ to posttest $(M=0.85, S D=0.39)$, and a main effect of feedback $[F(1$, $54)=8.08, p<.01]$ showed that slopes were larger with inflated feedback $(M=0.81, S D=0.39)$ than with no feedback $(M=0.65, S D=0.33)$. A Test $\times$ Feedback interaction $[F(1$, $\left.54)=30.09, p<.001, \eta_{\mathrm{p}}{ }^{2}=.36\right]$ showed that changes in slope from pretest to posttest depended on whether feedback was provided (see Fig. 1). However, all of these effects were qualified by a Test $\times$ Test Modality $\times$ Practice Modality $\times$ Feedback interaction $\left[F(1,54)=6.53, p<.05, \eta_{\mathrm{p}}{ }^{2}=.11\right]$. No other effects were significant (all other $p \mathrm{~s}>.18$ ).

To investigate the four-way interaction, separate 2 (test: pre vs. post) $\times 2$ (test modality: touch vs. audition) $\times 2$ (practice modality: touch vs. audition) ANOVAs were performed on slopes in the no-feedback and inflated-feedback conditions, respectively. In the no-feedback condition, a main effect of test modality $\left[F(1,26)=37.05, p<.001, \eta_{\mathrm{p}}{ }^{2}=.59\right]$ showed that slopes were larger in touch $(M=0.79, S D=$ $0.32)$ than in audition $(M=0.50, S D=0.29)$. No other effects were significant (all other $p \mathrm{~s}>.23$; see Fig. 1, top).

In the inflated-feedback condition, a main effect of test modality $\left[F(1,28)=27.27, p<.001, \eta_{\mathrm{p}}{ }^{2}=.49\right]$ showed that slopes were larger in touch $(M=0.95, S D=0.34)$ than in audition $(M=0.69, S D=0.39)$, and a main effect of test $\left[F(1,28)=92.30, p<.001, \eta_{\mathrm{p}}{ }^{2}=.77\right]$ showed that slopes increased from pretest $(M=0.61, S D=0.29)$ to posttest $(M=1.03, S D=0.37)$. However, these effects were qualified by a Test $\times$ Test Modality $\times$ Practice Modality interaction $\left[F(1,28)=7.93, p<.01, \eta_{\mathrm{p}}{ }^{2}=.22\right]$. Follow-up $t$ tests with Bonferroni corrections revealed that slopes increased from pretest to posttest for all test and practice modalities (all $p$ s $<.0125$; see Fig. 1, bottom).

Such results show that feedback on perception by audition recalibrated perception by audition and by touch, and 


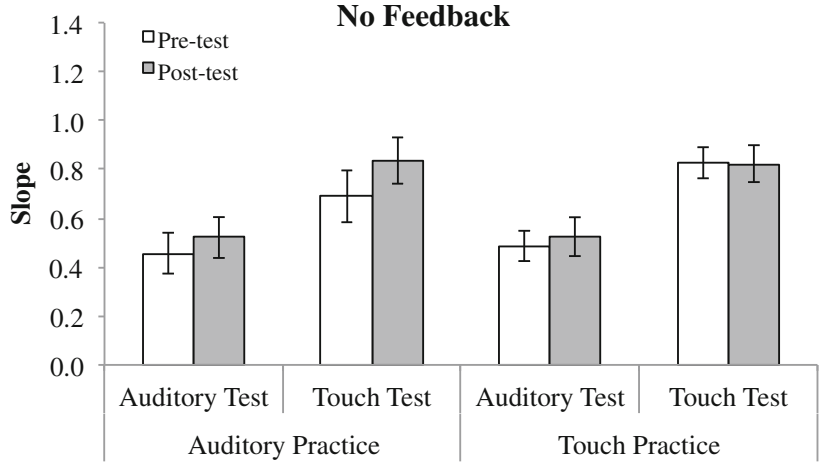

Feedback

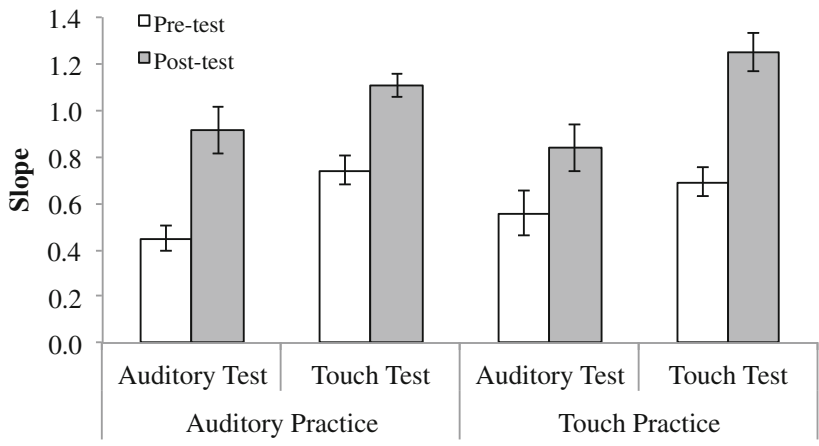

Fig. 1 Slope values for the test and practice modalities in the nofeedback (top) and inflated-feedback (bottom) conditions

feedback on perception by touch recalibrated perception by touch and by audition (see Fig. 1, bottom). To confirm that recalibration transferred in both directions, we analyzed the degree to which recalibration occurred in the untrained modalities. We conducted two separate 2 (test: pre vs. post) $\times 2$ (feedback: inflated feedback vs. no feedback) ANOVAs on slopes - one ANOVA on perception by audition with touch practice, and one on perception by touch with auditory practice. In each case, we found a Test $x$ Feedback interaction [audition with touch practice, $F(1,27)=6.65, p<.05, \eta_{\mathrm{p}}{ }^{2}=.20$; touch with audition practice, $F(1,27)=7.08, p<.05, \eta_{\mathrm{p}}{ }^{2}=.21$ ], providing direct evidence of recalibration and of transfer of recalibration (in both directions) (cf. Stephen \& Hajnal, 2011).

We also investigated whether the degree of recalibration in a given modality depended on the modality in which feedback was provided. For participants in the inflatedfeedback condition, we subtracted the pretest slope from the posttest slope for each test and practice modality. Follow-up $t$ tests with Bonferroni correction showed that (1) when feedback was provided on perception by touch, increases in slopes were larger for touch $(M=+0.55$, $S D=0.34)$ than for audition $(M=+0.28, S D=0.24)$ $[t(14)=3.03, p<.01]$, and (2) when feedback was provided on perception by audition, no differences emerged between the increases in slope for touch $(M=+0.36, S D=0.17)$ and for audition $(M=+0.46, S D=0.41)$ (see Fig. 2). Such

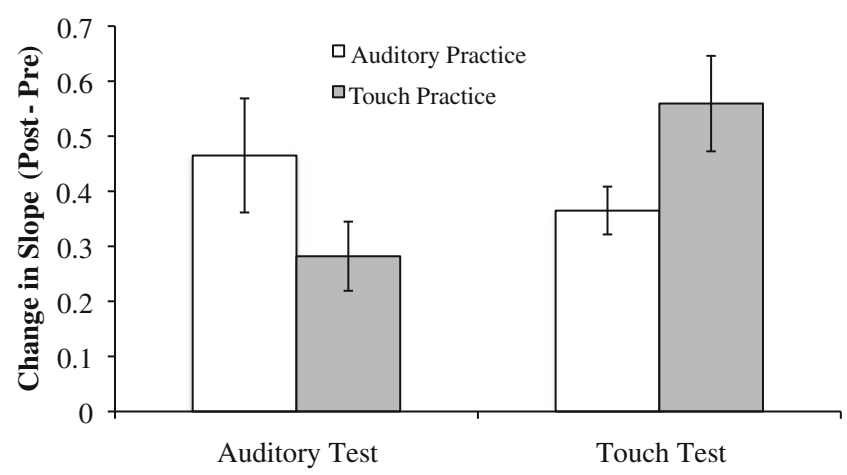

Fig. 2 Changes in slope (posttest - pretest) for the test and training modalities in the inflated-feedback condition

results show that feedback on perception by touch recalibrated perception by touch more than it did perception by audition, but that feedback on perception by audition recalibrated perception by touch and perception by audition equally.

A 2 (test: pre vs. post) $\times 2$ (test modality: touch vs. audition) $\times 2$ (practice modality: touch vs. audition $) \times 2$ (feedback: inflated feedback vs. no feedback) mixed-design ANOVA was also performed on the intercepts. A main effect of modality $\left[F(1,54)=92.30, p<.001, \eta_{\mathrm{p}}{ }^{2}=.64\right]$ showed that intercepts were smaller in touch $(M=-2.6 \mathrm{~cm}, S D=16.1)$ than in audition $(M=+20.6 \mathrm{~cm}, S D=20.3)$, and a Test $\times$ Test Modality interaction $\left[F(1,54)=11.27, p<.001, \eta_{\mathrm{p}}{ }^{2}=.17\right]$ showed that this difference was more pronounced in the posttest than in the pretest (see Fig. 3). Importantly, such effects were unaccompanied by effects of feedback, suggesting that the changes in offset error from pretest to posttest were independent of whether feedback was provided.

For such results to demonstrate transfer of recalibration, changes in perceived length from pretest to posttest must reflect a specific rescaling of perceived length to a detectable stimulation pattern learned via feedback. Therefore, it is necessary to rule out the possibility that such changes merely reflect a post-hoc decision to globally shift perceived lengths on the basis of a nonspecific rule (e.g., "too long" or "too

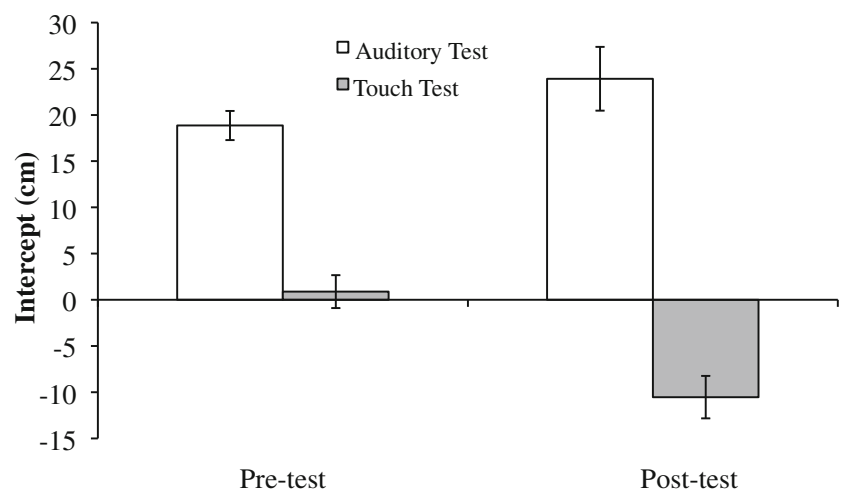

Fig. 3 Intercept values for the two test modalities in the pretest and posttest 
short") learned via feedback. Our finding that feedback resulted in changes in scaling but not offset error provides preliminary evidence to this end. However, stronger evidence would require demonstrating that following feedback (1) changes in perceived length from pretest to posttest differed for different rod lengths (i.e., such changes were not merely a global and nonspecific shifting of perceived length) and (2) the change in perceived length from pretest to posttest for a given rod was predicted by the error for that rod in the pretest (i.e., that such changes were specific rescalings on the basis of feedback) (cf. Wagman \& Abney, 2012).

To investigate the first question, we calculated difference scores by subtracting pretest perceived length from posttest perceived length for each rod for participants who received feedback. A 2 (test modality: audition vs. touch) $\times 2$ (practice modality: audition vs. touch) $\times 10$ (rod length) ANOVA was then conducted on these values. A main effect of rod length $\left[F(9,252)=17.76, p<.05, \eta_{\mathrm{p}}{ }^{2}=.39\right]$ confirmed that changes in perceived length from pretest to posttest were specific to rod length and were not merely due to a nonspecific shifting of perceived lengths.

To investigate the second question, we calculated two additional difference scores for the participants who received feedback. First, we calculated pretest "error" scores by subtracting the perceived length of each rod in the pretest from 1.5 times the length of that rod. $^{2}$ We also calculated "perceptual change" scores by subtracting the mean pretest perceived length from the mean posttest perceived length for each rod. In all conditions, we found significant positive correlations between these scores (with $r$ values ranging from +0.78 to +0.96 , all $p \mathrm{~s}<.001$ ). This confirms that the change in perceived length from pretest to posttest for a given rod was predicted by the amount of "error" on that rod in the pretest. In other words, feedback resulted in a specific rescaling of perceived length to actual length. Taken together, these results suggest that the changes in perceived length from pretest to posttest reflect a specific rescaling of perceived length to a detectable stimulation pattern learned via feedback.

\section{General discussion}

Previous research showing that recalibration of the perception of length transferred from audition to touch had provided preliminary evidence that calibration of the perception of length is modality-independent (Wagman \& Abney, 2012). In the present experiment, we provided a stronger test of this claim by investigating whether perception

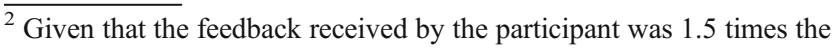
actual length of each rod, error was relative to this value, and not to the actual length of the rod.
}

transfers in both directions (i.e., from audition to touch, and vice versa). The new findings build on and expand those from the previous research. First, the perception of length by both touch and audition was systematically and linearly related to actual length, but perception by touch was calibrated better than perception by audition (Wagman \& Abney, 2012). Second, recalibration of perception occurred only when feedback was provided during practice (Wagman, Carello, Schmidt, \& Turvey, 2009; Wagman, McBride, \& Trefzger, 2008; Withagen \& Michaels, 2005). Importantly, such recalibration was shown to be a specific rescaling of perceived length on the basis of the feedback received, and not a post-hoc global shifting of perceived lengths. Finally, recalibration of the perception of length transferred in both directions - from audition to touch and from touch to audition - bolstering the claim that calibration of perception (of length) is modality-independent (or, at minimum, that calibration is not modality-specific; Wagman \& Abney, 2012).

\section{Calibration and modality-independent stimulation patterns}

These results contribute to an understanding of the nature of the stimulation pattern(s) that perceivers become calibrated to as result of feedback. Previous research has shown that recalibration of perception of a given property is not specific to a given effector (Stephen \& Hajnal, 2011; Withagen \& Michaels, 2004; but see Durgin, Fox, \& Kim, 2003) and that recalibration of perception by a given effector is not specific to a given property (Wagman \& Van Norman, 2011; Withagen \& Michaels, 2007). The results of the present experiment suggest that recalibration of the perception of a given property is not specific to a given modality (i.e., that recalibration is modality-independent). To the extent that this is the case, the stimulation pattern(s) that perceivers become calibrated to when they are provided with feedback should also be modality-independent. In the case of perception of length by touch and audition, it is likely that this stimulation pattern is anchored in the object's mechanical properties (i.e., mass, static moment, and moment of inertia). Such properties have the potential to structure both patterns of vibration when an object strikes a surface and muscular deformations when an object is wielded. Thus, such stimulation patterns may support the perception of length by both touch and audition, despite the anatomical, physiological, and functional differences between these modalities (Carello et al., 1998).

Perception and modality-independent stimulation patterns

The results also contribute to the understanding of the nature of the stimulation patterns that support perception in general. In particular, the findings are consistent with the proposal 
that such stimulation patterns are invariant and modalityindependent. According to this proposal, an object or event structures a given energy array such that the structure provides information about its source. Moreover, lawful relationships among the patterns of stimulation across energy arrays are such that structure in any one of these arrays can be sufficient to support perception (Carello, Wagman, \& Turvey, 2005; Rosenblum, 2004).

Importantly, however, the mere availability of modalityindependent stimulation patterns does not guarantee that the perception of a given property by means of different perceptual modalities will be equivalent. Although structure in one energy array may be sufficient to support perception of a given property, structure in another energy array may do so more optimally (see Rosenblum, 2004). Moreover, given differential lifetime experience perceiving a given property by different perceptual modalities, perceivers will likely be better attuned (and calibrated) to stimulation patterns in some energy arrays than in others (Wagman et al., 2009). Thus, despite the potential for modality-independent stimulation patterns, there are likely to be modality-dependent abilities to perceive a given property. Such modalitydependent abilities likely underlie differences in (1) the calibration of perception by touch and perception by audition (see Figs. 1 and 2) the transfer of recalibration from touch to audition, and vice versa (see Fig. 2).

Author note We thank Rachael Pinkerman for help with data collection. D.H.A. is now at the School of Social Sciences, Humanities and Arts, University of California, Merced.

\section{References}

Cabe, P. A., \& Wagman, J. B. (2010). Characterizing perceptual learning using regression statistics: Development of a perceptual calibration index. The American Journal of Psychology, 123, 253-267.

Carello, C., Anderson, K. L., \& Peck, A. (1998). Perception of object length by sound. Psychological Science, 9, 211-214. doi:10.1111/ 1467-9280.00040

Carello, C., Wagman, J. B., \& Turvey, M. T. (2005). Acoustic specification of object properties. In J. Anderson \& B. Anderson (Eds.), Moving image theory: Ecological considerations (pp. 79-104). Carbondale, IL: Southern Illinois University Press.

Durgin, F. H., Fox, L. F., \& Kim, D. H. (2003). Not letting the left leg know what the right leg is doing: Limb-specific locomotor adaptation to sensory-cue conflict. Psychological Science, 14, 567572. doi:10.1046/j.0956-7976.2003.psci 1466.x

Fitzpatrick, P., Carello, C., Schmidt, R. C., \& Corey, D. (1994). Haptic and visual perception of an affordance for upright posture. Ecological Psychology, 6, 265-287. doi:10.1207/s15326969 eco0604 2

Gibson, J. J. (1966). The senses considered as perceptual systems. Boston, MA: Houghton Mifflin.
Gordon, M. S., \& Rosenblum, L. D. (2004). Perception of soundobstructing surface using body-scaled judgments. Ecological Psychology, 16, 87-113.

Hajnal, A., Fonseca, S., Harrison, S., Kinsella-Shaw, J., \& Carello, C. (2007). Comparison of dynamic (effortful) touch by hand and foot. Journal of Motor Behavior, 39, 82-88. doi:10.3200/ JMBR.39.2.82-88

Palatinus, Z., Carello, C., \& Turvey, M. T. (2011). Principles of partwhole selective perception extend to the torso. Journal of Motor Behavior, 43, 87-93. doi:10.1080/00222895.2010.538767

Rieser, J. J., Pick, H. L., Ashmead, D. H., \& Garing, A. E. (1995). Calibration of human locomotion and models of perceptual-motor organization. Journal of Experimental Psychology. Human Perception and Performance, 21, 480-497. doi:10.1037/00961523.21.3.480

Rosenblum, L. D., \& Gordon, M. S. (2001). The generality of specificity: Some lessons from audiovisual speech. The Behavioral and Brain Sciences, 24, 239-240. doi:10.1017/S0140525X01503945

Rosenblum, L. D. (2004). Speech perception as a multimodal phenomenon, Current Directions in Psychological Science, 17, 405-409

Rosenblum, L. D., \& Robart, R. L. (2007). Hearing silent shapes: Identifying the shape of a sound-obstructing surface. Ecological Psychology, 4, 351-366. doi:10.1080/10407410701557844

Stephen, D., \& Hajnal, A. (2011). Transfer of calibration between hand and foot: Functional equivalence and fractal fluctuations. Attention, Perception, \& Psychophysics, 73, 1302-1328. doi:10.3758/s13414011-0142-6

Turvey, M. T., \& Carello, C. (2011). Obtaining information by dynamic (effortful) touch. Philosophical Transactions of the Royal Society B, 366, 3123-3132. doi:10.1098/rstb.2011.0159

Wagman, J. B., \& Abney, D. H. (2012). Transfer of recalibration from audition to touch: Modality independence as a special case of anatomical independence. Journal of Experimental Psychology. Human Perception and Performance, 38, 589-602. doi:10.1037/ a 0025427

Wagman, J. B., Carello, C., Schmidt, R. C., \& Turvey, M. T. (2009). Is perceptual learning unimodal? Ecological Psychology, 21, 37-67. doi:10.1080/10407410802626027

Wagman, J. B., McBride, D. M., \& Trefzger, A. J. (2008). Perceptual experience and posttest improvements in perceptual accuracy and consistency. Perception \& Psychophysics, 70, 1060-1067. doi:10.3758/PP.70.6.1060

Wagman, J. B., \& Van Norman, E. R. (2011). Transfer of calibration in dynamic touch: What do perceivers learn when they learn about length of a wielded object? Quarterly Journal of Experimental Psychology, 64, 889-901. doi:10.1080/17470218.2010.526233

Withagen, R., \& Michaels, C. F. (2004). Transfer of calibration in length perception by dynamic touch. Perception \& Psychophysics, 66, 1282-1292. doi:10.3758/BF03194998

Withagen, R., \& Michaels, C. F. (2005). The role of feedback information for calibration and attunement in perceiving length by dynamic touch. Journal of Experimental Psychology. Human Perception and Performance, 31, 1379-1390. doi:10.1037/ 0096-1523.31.6.1379

Withagen, R., \& Michaels, C. F. (2007). Transfer of calibration between length and sweet-spot perception by dynamic touch. Ecological Psychology, 19, 1-19. doi:10.1080/10407410709336948

Withagen, R., \& van Wermeskerken, M. (2009). Individual differences in learning to perceive length by dynamic touch: Evidence for variation in perceptual learning capacities. Attention, Perception, \& Psychophysics, 71, 64-75. doi:10.3758/ APP.71.1.64 